\title{
Optimal placement of PMU for complete observability of the interconnected power network considering zero-injection bus: A numerical approach
}

\author{
Rohit Babu', Biplab Bhattacharyya ${ }^{2}$ \\ ${ }^{1}$ Department of Electrical and Electronics Engineering, Bharat Institute of Engineering and Technology, India \\ ${ }^{2}$ Department of Electrical Engineering, Indian Institute of Technology (Indian School of Mines), India
}

\begin{tabular}{l} 
Article Info \\
\hline Article history: \\
Received Sep 12, 2019 \\
Revised Feb 19, 2020 \\
Accepted Mar 3, 2020 \\
\hline Keywords: \\
Bus observability redundancy \\
index \\
Interconnected power network \\
Measurement redundancy \\
Observability analysis \\
Optimal PMU placement \\
Phasor measurement unit \\
Total system observability \\
redundancy index
\end{tabular}

\section{Corresponding Author:}

Rohit Babu,

Department of Electrical and Electronics Engineering,

Bharat Institute of Engineering and Technology,

Mangalpally, Ibrahimpatnam, Hyderabad-501510, India.

Email: rohitbabu@biet.ac.in

\begin{abstract}
This paper presents an approach to place the phasor measurement unit (PMU) optimally, which minimizes the setup cost of PMU. This methodology attains complete state estimation of the interconnected power networks. An integer linear programming (ILP) method is explored for the optimal PMU placement problem. It is used to determine the optimal location and minimum number of PMUs necessary to make the interconnected power network completely observable. ILP may provide many solutions if acquainting buses to zero injection buses are unhandled. In the case of more than one solution, a bus observability redundancy index and total system observability redundancy index is proposed to find the most promising solutions set for redundancy measurement. The proposed algorithm is applied to benchmark the optimal PMU placement solutions for the IEEE 14-bus, IEEE 30-bus, New England 39-bus, IEEE 118-bus, and NRPG 246-bus test systems. The obtained results of the proposed approach are compared with the existing standard algorithm, and it is observed that the proposed approach achieves complete observability of the interconnected power network under base-load conditions.
\end{abstract}

This is an open access article under the CC BY-SA license.

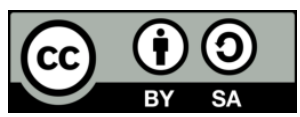

\section{INTRODUCTION}

Interconnected power network measurement, synchronized on a universal basis, is shifting from the laboratory to the utility. PMU-an instrument that uses global positioning satellite (GPS) technology, provides novel opportunities for the interconnected power network monitoring, observability, control, and protection $[1,2]$. In recent years, supervisory control and data acquisition (SCADA) systems are used for surveillance of the network conditions, but it gives unsynchronized measurements dominating the inconsistent estimation of the power network states [3]. Furthermore, a scan rate of data (2-4 samples per cycle) makes the SCADA system inefficient for measuring the dynamic/transient behavior occurring in the power network. These issues can be vanquished by the advent of PMUs, which mitigate this problem by using GPS technology and makes precise measurements of the network states [4]. The PMU with a faster scan rate (25 samples per second) makes itself acceptable for the observability of the power networks. Although the placement of PMU at each bus in the interconnected power network would provide all the states of the network system, it is injudicious as PMU and its communication facilities are expensive. Thus, an appropriate methodology is necessary for the site selection of PMUs. PMU may measure voltage 
and current phasors and covers other characteristics such as preservative actions. The goal of the present paper is restricted to find the minimum number and the optimal location of PMU for complete observability of the interconnected power network states under intact conditions.

An interconnected power network is said to be fully observable only when all of its states are uniquely measured [5-7]. The vigorous research activities on the issues of finding the minimum number and optimal locations of PMU have already been published in the open literature. Phadke et al. do pioneering work in the field of PMU development and its application in the mid-80s [2, 8]. Some scientists, engineers, and researchers believe that the deployment of PMU at each bus will escort to a simplified linear state estimator. However, later this problem is resolved in [1], as each PMU can measure not only the bus voltage but also the branch current incident to the bus. Hence, proper site selection of PMU can make the power network completely observable. In [9 and 10], a novel binary search algorithm is used to find the minimum number and optimal locations of PMU for interconnected power network state estimations. In [11], the authors used a binary particle swarm optimization technique for finding the optimal locations of PMU. In [12], a novel intelligent search-based technique for the placement of PMUs in connected power networks while maintaining complete observability is proposed.

For the optimal placement of PMU, a genetic algorithm-based procedure is developed in [13]. In $[14,15]$, the authors proposed a topologically based three-stage optimal PMU placement (OPP) approach for the observability of interconnected power networks. In [16], the authors suggested a novel investment decision model for finding the optimal location of PMUs that gives assurance of the complete observability of the power grid. An ILP and multi-criteria decision-making based approach is proposed in [17] for placing the PMUs in multiple stages over a given period that guarantees fully interconnected power network observability even during a line outage or a PMU collapse. In [18, 19], authors used a multi-objective biogeography based optimization algorithm for site selection of PMU which makes the power network fully observable. In $[20,21]$, the authors used integer programming to find the minimum number and optimal locations of PMU for state estimation. In [22, 23], the problem related to OPP and conventional power flow measurements to assure observability during faulted conditions in power networks are considered. In that, at the beginning, the methodology is presented as a nonlinear integer programming problem and then changed into a similar ILP problem through Boolean suggestions. An integer programming based methodology is used by the authors in $[24,25]$ for the OPP problem in the interconnected power network. In [26], the authors explored the consequences of channel capacity of PMUs on their optimal locations to ensure that the interconnected power network is fully observable. In [27], the authors proposed a new methodology for the OPP problem in a connected power network that is suffering from random component outages. In [28], the authors proposed the sum of the variance of the robust estimators to determine the PMU placement problem. Also, the placements obtained are further illustrated based on the variance of the estimated states. Both the weighted least squares and robust estimators are taken into consideration. The OPP problem is evaluated as a binary semidefinite programming model with binary decision variables in [29]. Both single PMU and line outage is considered.

Two deterministic formulations are proposed in [30], which are mixed-integer linear programming and nonlinear programming, for solving the OPP problem to achieve complete power network observability. The authors have proposed a novel combinatorial formulation for monitoring the complete power grid in [31]. A multi-criteria decision support method, analytical hierarchy process, has been used to solve the OPP problem. The Pareto approach by nondominated sorting genetic algorithm II is proposed in [32] to minimize the PMU placement cost with the current channel selection and the state estimation error. In [33, 34], the two-phase branch-and-bound algorithm is proposed for unraveling the OPP problem. The main contribution of the presented work is to exclude radial buses (RBs) from the list of potential locations for employing a PMU because a PMU located at a radial bus can measure the voltage phasors at that bus and only one additional bus which is associated to it. PMU installed at a bus linked with the RB can measure the voltage phasor of the radial bus by using the measurement of the current phasor through the radial line. Therefore, a PMU is pre-assigned to each bus connected to a radial bus.

In this paper, we propose an integer linear programming algorithm for finding the minimum number and optimal locations of PMUs for the observability of the interconnected power network states. A different methodology that is numerical and uses integer programming is conferred. This method enables the unchallenging investigation of power network observability for mixed measurement sets. The developed criterion has removed any redundancies in PMU placement attained from the proposed algorithm. The proposed method is applied on IEEE 14-bus [35], IEEE 30-bus [35], New England (NE) 39-bus [36], IEEE 118-bus [35], and northern regional power grid (NRPG) 246-bus [37] test systems. The results obtained with the help of the proposed approach have been compared with the existing standard results published in the open literature to ensure the efficacy of the proposed method in OPP issues. The remaining paper is 
divided into the following segments: Section 2 explains the research methodology. In section 3, case studies and details of results are given, and in section 4, the conclusion of the paper is presented.

\section{RESEARCH METHOD}

PMU placed at a specific bus in power network precisely measures the voltage phasor of the bus and the phasor currents for all branches incident to that bus [9]. Rely on the kind of PMUs used, the number of channels used for estimating voltage phasor and current phasor will differ. Therefore, the complete power network observability can be achieved by placing a PMU at a specific bus in the system. The main goal of the OPP problem is to furnish an observable network by using a minimum number and optimal locations of PMUs [38]. For the graphical representation of the OPP, IEEE 14-bus system is shown in figure 1 as an example [39]. In the interconnected power network, there is a bus known as a zero-injection bus (ZIB) with no generation and no loads connected to it. This ZIB minimizes the number of required PMUs by one because it can be merged with one of its adjacent buses. This means that the network topology is modified by using the merging process. It needs to redefine the network equations to reflect these changes [40].

In IEEE 14-bus system, three PMUs are located at $\mathrm{B}_{2}, \mathrm{~B}_{6}$, and $\mathrm{B}_{9} . \mathrm{B}_{7}$ is only a zero injection bus (ZIB). The PMU at $B_{2}$ not only measure the phase voltage of $B_{2}$, but also the phase current of $L_{1}, L_{2}, L_{3}$ and $\mathrm{L}_{4}$. The phase voltage at $\mathrm{B}_{1}, \mathrm{~B}_{3}, \mathrm{~B}_{4}$ and $\mathrm{B}_{5}$ can be acquired from the line currents by using Ohm's law. After finding phase voltages at $\mathrm{B}_{1}, \mathrm{~B}_{2}, \mathrm{~B}_{3}, \mathrm{~B}_{4}$ and $\mathrm{B}_{5}$, the phase current of $\mathrm{L}_{4}, \mathrm{~L}_{6}$ and $\mathrm{L}_{7}$ are determined. Similarly, PMU at $\mathrm{B}_{6}$ measures phase voltage at $\mathrm{B}_{6}$ and the phase currents of $\mathrm{L}_{8}, \mathrm{~L}_{14}, \mathrm{~L}_{15}$ and $\mathrm{L}_{16}$ are also measured. Again by using Ohm's law, the phase voltages at $\mathrm{B}_{5}, \mathrm{~B}_{11}, \mathrm{~B}_{12}$, and $\mathrm{B}_{13}$ can be measured and phase current of $\mathrm{L}_{19}$ can be determined. Further, PMU at $\mathrm{B}_{9}$ can measure the phase voltage at $\mathrm{B}_{9}$ and phase currents of $\mathrm{L}_{11}, \mathrm{~L}_{12}, \mathrm{~L}_{13}$ and $\mathrm{L}_{17}$ and by applying Ohm's law, the phase voltages at $\mathrm{B}_{4}, \mathrm{~B}_{7}, \mathrm{~B}_{10}$ and $\mathrm{B}_{14}$ can be evaluated. In addition, the phase current of $\mathrm{L}_{9}$ is also determined. As a result, phase voltages of $\mathrm{B}_{10}, \mathrm{~B}_{11}, \mathrm{~B}_{13}$ and $\mathrm{B}_{14}$ are known, phase currents of $\mathrm{L}_{18}$ and $\mathrm{L}_{20}$ are also estimated. By using phase currents of $\mathrm{L}_{9}$ and $\mathrm{L}_{12}$, the phase current of $\mathrm{L}_{10}$ can also be evaluated by using Kirchhoff's current law (KCL). The phase voltage at a radial bus, $B_{8}$, can now be determined by utilizing the phase voltage at $B_{7}$ and the phase current of $L_{10}$. Hence, the complete IEEE 14-bus system is observable by placing PMUs at $\mathrm{B}_{2}, \mathrm{~B}_{6}$, and $\mathrm{B}_{9}$ and considering $\mathrm{B}_{7}$ as a $\mathrm{ZIB}$.

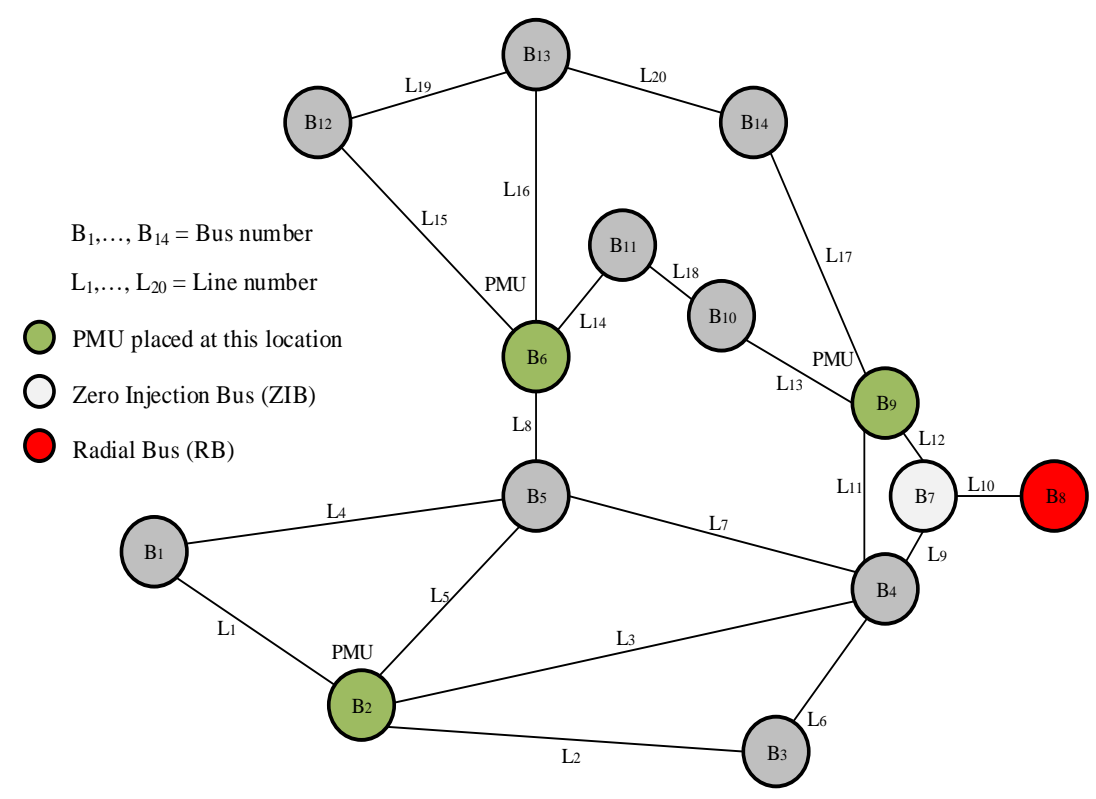

Figure 1. Optimal placement of PMU for IEEE 14-bus test system [39]

\subsection{ILP based PMU placement method}

The integer programming (IP) is a numerical optimization programming for issues having integer variables, and it is the most common method for unraveling the OPP problem. IP is mentioned as integer linear programming (ILP) when the constraints and objective function are linear. In an ILP, when some variables are integers and other non-integers, then ILP is mentioned as mixed-ILP (MILP). In case

\footnotetext{
Optimal placement of PMU for complete observability of the interconnected power network... (Rohit Babu)
} 
the variables are confined within $[0,1]$ then ILP can be treated as binary- ILP (BILP) technique. Therefore, the constraints played a significant role when using the ILP method to unravel the OPP issue.

\subsubsection{OPP problem formulation}

The objective of the OPP is to obtain the minimum number of PMUs needed and their locations for achieving full observability of the interconnected power network. Thus, the OPP is formulated as follows:

$$
\begin{aligned}
& \min \sum_{k=1}^{n} c_{k} \cdot y_{k} \\
& \text { s.t. } a(Y) \geq b \\
& Y=\left[\begin{array}{lll}
y_{1} & y_{2} & y_{3}
\end{array} \ldots y_{n}\right]^{T} \\
& y_{k} \in\{0,1\}
\end{aligned}
$$

where, $n$ is the number of buses in interconnected power network for the deployment of PMUs, $C_{k}$ is the cost of PMU set-up at $k^{\text {th }}$ bus, $Y$ is the binary decision variable vector having element $y_{k}$ which decides achievability of PMU on $k^{\text {th }}$ bus and whose entries are defined as in (3). $\alpha(Y)$ are the observability constraints whose entries are non-zero if the bus voltage is noticeable w.r.t. the given sets of measurement and zero otherwise.

$$
y_{k}= \begin{cases}1, & \text { if } P M U \text { is set }- \text { up at } k^{\text {th }} \text { bus } \\ 0, & \text { otherwise }\end{cases}
$$

The entries in a are as follows:

$$
a_{k, m}=\left\{\begin{array}{lr}
1, \text { if bus } k \text { is linked to bus } m \\
1, & \text { if } k=m \\
0, & \text { otherwise }
\end{array}\right.
$$

and

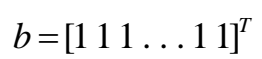

The methodology for developing the constraint equation is explained for two attainable conditions (1) when there are no conventional measurements or ZIBs and (2) considering ZIBs [39]. The IEEE 14-bus test system is taken as an example to describe the above mentioned cases.

Case 1: A system with no conventional measurements.

In this case, ZIBs are ignored. For the sake of building the constraint set, the binary connectivity matrix $a$, as defined in (4), is constituted first.st. The $a(n \times n)$ matrix for the IEEE 14-bus test system is given in (6).

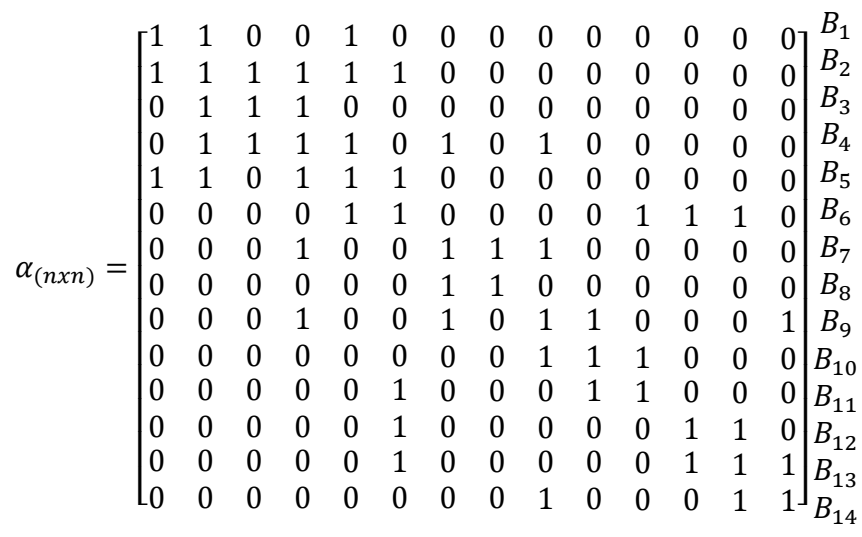

The constraints for this condition can be developed as (7). 


$$
f(Y)=a . Y=\left\{\begin{array}{lr}
f_{1}=y_{1}+y_{2}+y_{5} & \geq 1 \\
f_{2}=y_{1}+y_{2}+y_{3}+y_{4}+y_{5} & \geq 1 \\
f_{3}=y_{2}+y_{3}+y_{4} & \geq 1 \\
f_{4}=y_{2}+y_{3}+y_{4}+y_{5}+y_{7}+y_{9} \geq 1 \\
f_{5}=y_{1}+y_{2}+y_{4}+y_{5}+y_{6} & \geq 1 \\
f_{6}=y_{5}+y_{6}+y_{11}+y_{12}+y_{13} & \geq 1 \\
f_{7}=y_{4}+y_{7}+y_{8}+y_{9} & \geq 1 \\
f_{8}=y_{7}+y_{8} & \geq 1 \\
f_{9}=y_{4}+y_{7}+y_{9}+y_{10}+y_{14} & \geq 1 \\
f_{10}=y_{9}+y_{10}+y_{11} & \geq 1 \\
f_{11}=y_{6}+y_{10}+y_{11} & \geq 1 \\
f_{12}=y_{6}+y_{12}+y_{13} & \geq 1 \\
f_{13}=y_{6}+y_{12}+y_{13}+y_{14} & \geq 1 \\
f_{14}=y_{9}+y_{13}+y_{14} & \geq 1
\end{array}\right.
$$

The operator ' + ' represents the logical 'OR' and the benefit of 1 in the right-hand side of the inequality assures that not less than one of the variables present in the sum will be non-zero; e.g. consider the constraint related with $\mathrm{B}_{1}$ and $\mathrm{B}_{2}$ as stated in (8).

$$
\begin{aligned}
& f_{1}=y_{1}+y_{2}+y_{5} \quad \geq 1 \\
& f_{2}=y_{1}+y_{2}+y_{3}+y_{4}+y_{5} \geq 1
\end{aligned}
$$

The constraint $f_{1} \geq 1$ signifies that a PMU must be installed either at $\mathrm{B}_{1}, \mathrm{~B}_{2}$ or $\mathrm{B}_{5}$ to observed $\mathrm{B}_{1}$. Likewise, the constrain $f_{2} \geq 1$ shows that a PMU must be installed at any one of the $\mathrm{B}_{1}, \mathrm{~B}_{2}, \mathrm{~B}_{3}, \mathrm{~B}_{4}$ or $\mathrm{B}_{5}$ so that $\mathrm{B}_{2}$ becomes observable.

Case 2: A system with zero injection (ZI) measurements.

In this case, we contemplate the exceedingly common position where zero injection measurements may exist but insufficient to make the interconnected power network completely observable. Again, the IEEE 14-bus test system, as shown in Figure 2 is considered, where $B_{7}$ is a ZIB. It is obvious to identify that if the phase voltages at each three out of the four, $\mathrm{B}_{4}, \mathrm{~B}_{7}, \mathrm{~B}_{8}$ and $\mathrm{B}_{9}$ are acknowledged, then the fourth one can be determined by using $\mathrm{KCL}$ at $\mathrm{B}_{7}$ where the total sum of the injected current is known. Therefore, the constraints associated with the ZI buses are to adapt with the acquainting buses of theses buses and build a set of non-linear constraints and this is concluded as depicted in Figure 2.

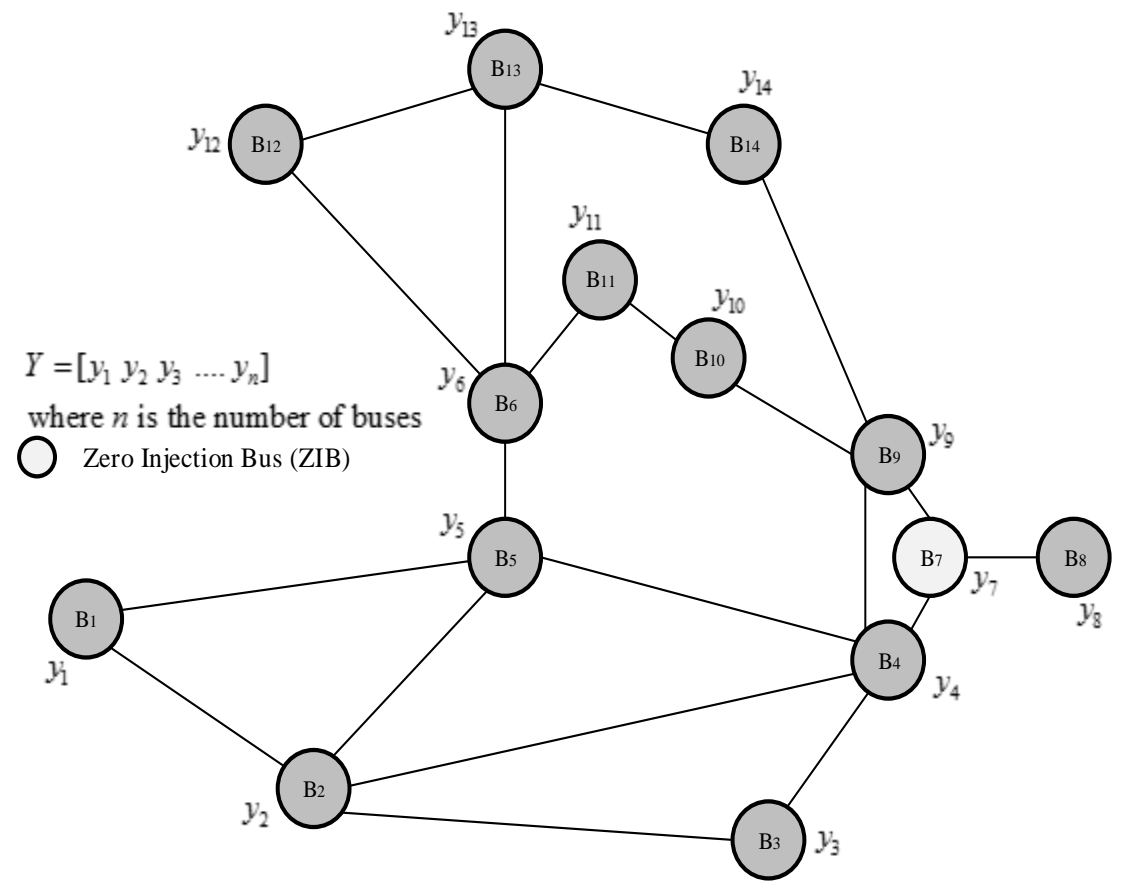

Figure 2. IEEE 14-bus test system with conventional measurements

\footnotetext{
Optimal placement of PMU for complete observability of the interconnected power network... (Rohit Babu)
} 
In IEEE 14-bus test system, the constraints associated with ZIB $\mathrm{B}_{7}$ with its acquainting buses $\mathrm{B}_{4}, \mathrm{~B}_{8}$, and $\mathrm{B}_{9}$ are altered as given in (9);

$$
\begin{array}{lr}
f_{4}=y_{2}+y_{3}+y_{4}+y_{5}+y_{7}+y_{9}+f_{7} \cdot f_{8} \cdot f_{9} \geq 1 \\
f_{8}=y_{7}+y_{8}+f_{4} \cdot f_{7} \cdot f_{9} & \geq 1 \\
f_{9}=y_{4}+y_{7}+y_{9}+y_{10}+y_{14}+f_{4} \cdot f_{7} \cdot f_{8} & \geq 1
\end{array}
$$

The operator '.' represents the logical 'AND' in (9). The explanations for $f_{i}$ are clear by utilizing the following features of the logical AND (.) and OR (+) operators. $A+B=B$ and $A . B=A$. Substituting for $f_{7}$ from (7) in $f_{4}$ of (9), yields:

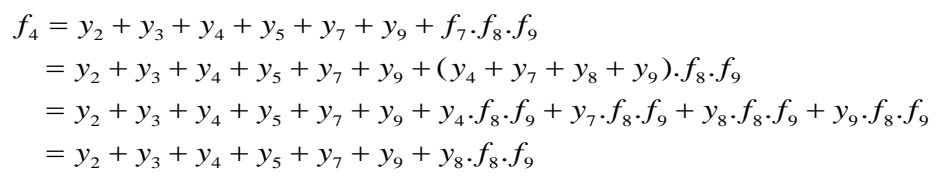

In (10), $y_{4} . f_{8} . f_{9}$ is removed as it is the subset of $y_{4}$. Similarly, $y_{7} . f_{8} . f_{9}$ and $y_{9} . f_{8} . f_{9}$ are also eliminated. It is observed that the explanation for $f_{7}$ should also indulge an ancillary product term given by $f_{4} \cdot f_{8} \cdot f_{9}$, however this term will be dilapidated. In each simulated condition, this estimation is found ineffective in the optimization process. Further, substituting for $f_{8}$ in (10), gives:

$$
\begin{aligned}
f_{4} & =y_{2}+y_{3}+y_{4}+y_{5}+y_{7}+y_{9}+y_{8} \cdot f_{8} \cdot f_{9} \\
& =y_{2}+y_{3}+y_{4}+y_{5}+y_{7}+y_{9}+y_{8} \cdot\left(y_{7}+y_{8}\right) \cdot f_{9} \\
& =y_{2}+y_{3}+y_{4}+y_{5}+y_{7}+y_{9}+y_{8} \cdot f_{9}
\end{aligned}
$$

again, substituting for $f_{9}$ in (11) yields:

$$
\begin{aligned}
f_{4} & =y_{2}+y_{3}+y_{4}+y_{5}+y_{7}+y_{9}+y_{8} \cdot f_{9} \\
& =y_{2}+y_{3}+y_{4}+y_{5}+y_{7}+y_{9}+y_{8} \cdot\left(y_{4}+y_{7}+y_{9}+y_{10}+y_{14}\right) \\
& =y_{2}+y_{3}+y_{4}+y_{5}+y_{7}+y_{9}+y_{8} \cdot y_{10}+y_{8} \cdot y_{14}
\end{aligned}
$$

similarly, implementing the same logic to other expressions, the constraint set can be written as (13).

$$
\begin{array}{lr}
f_{4}=y_{2}+y_{3}+y_{4}+y_{5}+y_{7}+y_{9}+y_{8} \cdot y_{10}+y_{8} \cdot y_{14} \quad \geq 1 \\
f_{8}=y_{4}+y_{7}+y_{8}+y_{9} & \geq 1 \\
f_{9}=y_{4}+y_{7}+y_{9}+y_{10}+y_{14}+y_{2} \cdot y_{8}+y_{3} \cdot y_{8}+y_{5} \cdot y_{8} & \geq 1
\end{array}
$$

It is observed that the constraints analogous to each bus remain unchanged, as shown in (7). But the constraint for B7 where the injection is measured is removed from the constraint set as the constraints related to ZIBs are incidentally considered by the product terms to add to the constraints related to the acquainting buses. The constraint for this condition is given as (14).

$$
f(Y)=\left\{\begin{array}{lr}
f_{1}=y_{1}+y_{2}+y_{5} & \geq 1 \\
f_{2}=y_{1}+y_{2}+y_{3}+y_{4}+y_{5} & \geq 1 \\
f_{3}=y_{2}+y_{3}+y_{4} & \geq 1 \\
f_{4_{-} \text {new }}=y_{2}+y_{3}+y_{4}+y_{5}+y_{7}+y_{9}+y_{8} \cdot y_{10}+y_{8} \cdot y_{14} & \geq 1 \\
f_{5}=y_{1}+y_{2}+y_{4}+y_{5}+y_{6} & \geq 1 \\
f_{6}=y_{5}+y_{6}+y_{11}+y_{12}+y_{13} & \geq 1 \\
f_{7}=y_{4}+y_{7}+y_{8}+y_{9} & \geq 1 \\
f_{8_{-} \text {new }}=y_{4}+y_{7}+y_{8}+y_{9} & \geq 1 \\
f_{9_{-} \text {new }}=y_{4}+y_{7}+y_{9}+y_{10}+y_{14}+y_{2} \cdot y_{8}+y_{3} \cdot y_{8}+y_{5} \cdot y_{8} & \geq 1 \\
f_{10}=y_{9}+y_{10}+y_{11} & \geq 1 \\
f_{11}=y_{6}+y_{10}+y_{11} & \geq 1 \\
f_{12}=y_{6}+y_{12}+y_{13} & \geq 1 \\
f_{13}=y_{6}+y_{12}+y_{13}+y_{14} & \geq 1 \\
f_{14}=y_{9}+y_{13}+y_{14} & \geq 1
\end{array}\right.
$$




\subsubsection{Algorithmic steps to obtain OPP problem}

To find the solution for integer linear/non-linear programming problem, TOMLAB/MINLP with MATLAB software package is used [41]. The implementation of the proposed algorithm to obtain OPP for an interconnected power network consisting ' $n$ ' no. of buses using TOMLAB/MINLP is explained as follows [42]:

Step 1. Obtain binary connectivity matrix $a(n \times n)$ using (4).

Step 2. Define $b_{-} l$ (lower bound on $\left.(y, a \cdot y)\right)$ and $b_{-} u$ (upper bound on $(y, a \cdot y)$ ).

Step 3. Explain ' $c$ ', cost of the installed PMUs at $k^{\text {th }}$ bus.

Step 4. Expound ' $y_{-} l, y_{-} u$ ', bound on variables.

Step 5. Initialize ' $y$ ' vector $\left(y_{-} 0\right)$, if empty set as 0 .

Step 6. Define' IntVars', each variable should be integer. This field is explained individually depending on the length. Variable indices should be in between $[1, \ldots, n]$.

Step 7. Explain ' $f I P^{\prime}$ ', upper bound of $f(y)$. Only consider solutions $<f I P$-epsilon, default $1 e 20$.

Step 8. Interpret the priorities of the integer variables 'VarWeight'. Can be any values, but lower values means higher priority.

Step 9. Assign routine for defining IP problem 'Pr $o b=$ mipAssign(step $1-$ to - step8)' .

Step 10. Display PMU locations ' $N_{P M U}$ ' using tomRun, the universal driver routine.

\subsubsection{Bus observability index (BOI)}

After obtaining the minimum number and optimal locations of PMUs, an expression for BOI is given as [39]. We can acknowledge BOI as a performance symbol at all aspects of optimization.

$$
B O I=a_{(n \times n)} \cdot N_{P M U}^{T}
$$

\subsubsection{Total system observability redundancy index (TSORI)}

The TSORI, which is the sum of bus observability for all buses, is given as follows [39]:

$$
T S O R I=\sum_{i=1}^{N_{P M U}} a_{(n \times n)} \cdot N_{P M U}^{T}
$$

where, $N_{P M U}$ is the total optimal number of PMUs obtained from step 10 of Section 3.2, $a$ is the binary connectivity matrix which is obtained from (4). As shown in (15) and (16) gives the BOI and TSORI for all the possible results of optimal locations of PMUs.

\section{RESULTS AND DISCUSSION}

The proposed approach for finding the minimum number and optimal locations of PMU has been applied on standard IEEE 14-bus, IEEE 30-bus, New England 39-bus, IEEE 118-bus and NRPG 246-bus test systems. Figure 3, shows the single line diagram of the NRPG 246 bus test system. TOMLAB/MINLP with MATLAB software package is used to perform the integer linear programming problem, as described in subsection 2.1.2. The technical configuration of the computer is Intel core: I3-2330M (2.2 GHz), L3 Cache: 3MB, and System Memory: 3GB DDR3 which is used for the simulation purpose. Table 1 shows the number and locations of zero injection buses (ZIBs) and radial buses (RBs) for the standard test systems. The proposed approach eliminates the RBs from the possible locations of PMUs. As a result, buses next to these RBs with higher branch connectivity are preferred as the PMU placement sites [10, 39]. Table 2 shows the buses where PMU is essentially placed in order to attain the observability of the RBs. Table 3 shows the minimum number of PMUs necessary to make the interconnected power network completely observable under usual operating states with computational time. Tables 4 and 5 shows the redundancy measurement for the standard test system ignoring and considering ZIBs.

The bus numbers are designated as $B_{1}, B_{2}, B_{3}, \ldots, B_{n}$ where $n=1,2,3, \ldots, N$. The entries in the tables symbolize the number of times a bus phase angle is measured by the optimal locations of PMUs. For example, in the case of IEEE 14-bus system, ignoring ZIBs, $B_{4}$ is observed three times. $B_{5}, B_{7}, B_{9}$ are observed two times and the rest of the buses are observed once. When considering ZIBs, $B_{4}$ and $B_{5}$ are observed two times and the remaining buses are observed once. Similarly, for the other test systems, redundancy measurement is shown.

Optimal placement of PMU for complete observability of the interconnected power network... (Rohit Babu) 


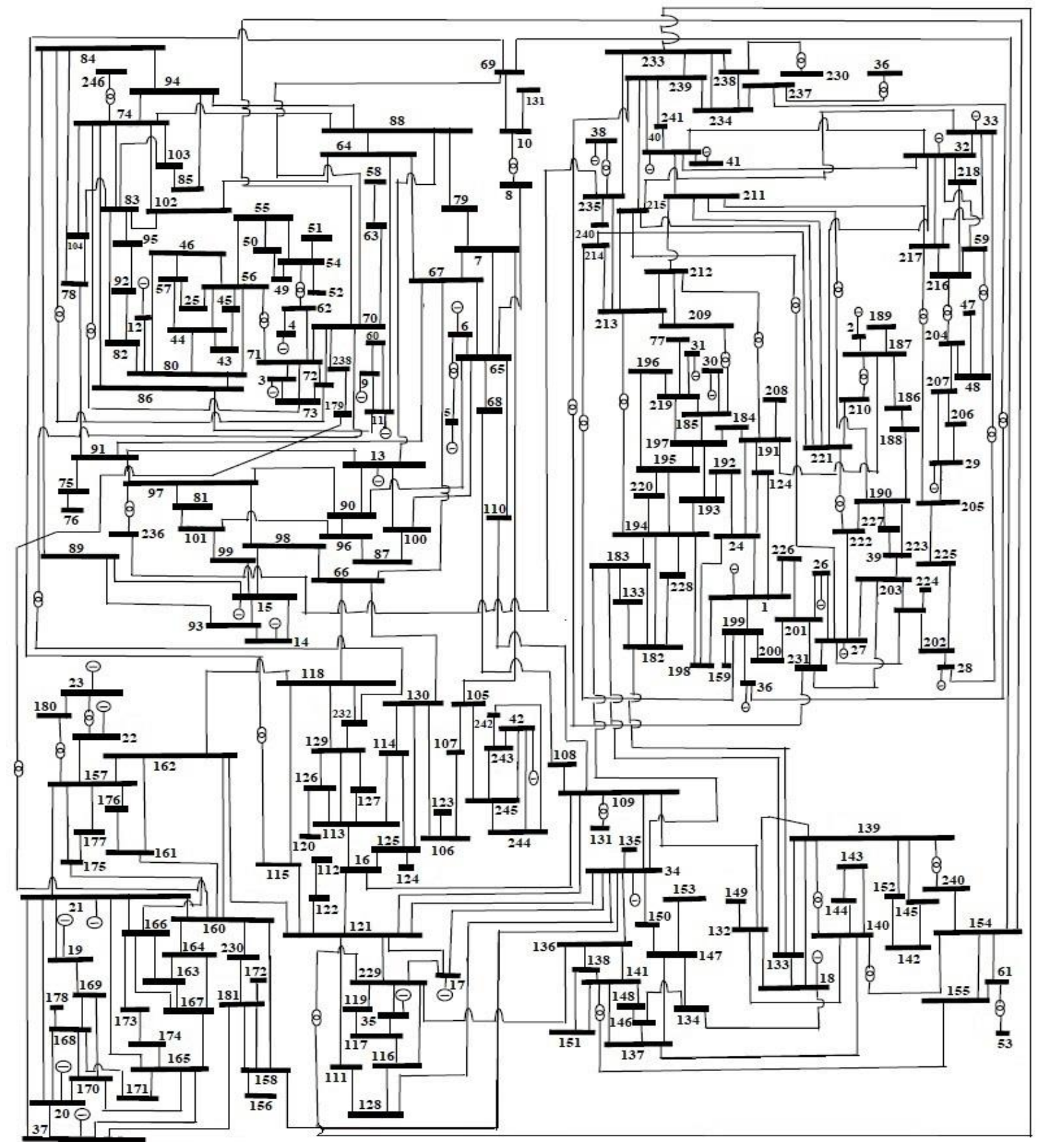

Figure. 3 Single line diagram of the NRPG 246 bus test system [37]

Table 1. Location of zero injection buses (zibs) and radial buses (RBs)

\begin{tabular}{|c|c|c|c|c|c|}
\hline $\begin{array}{l}\text { Test } \\
\text { system }\end{array}$ & $\begin{array}{l}\text { Size (buses, } \\
\text { lines ) }\end{array}$ & $\begin{array}{l}\text { \# of } \\
\text { ZIBs }\end{array}$ & ZIBs & $\begin{array}{l}\text { \# of } \\
\text { RBs }\end{array}$ & RBs \\
\hline 14-bus & 14,20 & 01 & $B_{7}$ & 01 & $B_{8}$ \\
\hline 30-bus & 30,41 & 06 & $B_{6}, B_{9}, B_{22}, B_{25}, B_{27}, B_{28}$ & 03 & $B_{11}, B_{13}, B_{26}$ \\
\hline 39-bus & 39,46 & 11 & $B_{1}, B_{2}, B_{5}, B_{6}, B_{9}, B_{11}, B_{13}, B_{14}, B_{17}, B_{19}, B_{22}$ & 09 & $\begin{array}{l}B_{30}, B_{31}, B_{32}, B_{33}, B_{34}, B_{35}, B_{36}, B_{37} \\
B_{38}\end{array}$ \\
\hline 118-bus & 118,186 & 10 & $B_{5}, B_{9}, B_{30}, B_{37}, B_{38}, B_{63}, B_{64}, B_{68}, B_{71}, B_{81}$ & 07 & $B_{10}, B_{73}, B_{87}, B_{111}, B_{112}, B_{116}, B_{117}$ \\
\hline 246-bus & 246,376 & 58 & $\begin{array}{l}B_{54}, B_{56}, B_{59}, B_{61}, B_{62}, B_{63}, B_{69}, B_{70}, B_{71}, B_{72}, \\
B_{73}, B_{74}, B_{75}, B_{80}, B_{81}, B_{86}, B_{102}, B_{103}, B_{104}, \\
B_{107}, B_{122}, B_{126}, B_{129}, B_{131}, B_{147}, B_{154}, B_{155}, \\
B_{167}, B_{175}, B_{179}, B_{180}, B_{183}, B_{209}, B_{210}, B_{211}, \\
B_{212}, B_{213}, B_{214}, B_{215}, B_{216}, B_{217}, B_{221}, B_{222}, \\
B_{226}, B_{229}, B_{230}, B_{231}, B_{232}, B_{233}, B_{234}, B_{236} \\
B_{237}, B_{238}, B_{239}, B_{240}, B_{241}, B_{243}, B_{244}\end{array}$ & 31 & $\begin{array}{l}B_{2}, B_{4}, B_{5}, B_{12}, B_{30}, B_{31}, B_{38}, B_{41}, B_{47}, \\
B_{51}, B_{52}, B_{53}, B_{58}, B_{76}, B_{77}, B_{112}, B_{120}, \\
B_{123}, B_{124}, B_{135}, B_{149}, B_{153}, B_{156}, B_{159}, \\
B_{172}, B_{178}, B_{189}, B_{208}, B_{224}, B_{246}\end{array}$ \\
\hline
\end{tabular}


Table 2. Pre-allocation of PMUs

\begin{tabular}{lll}
\hline Test system & \multicolumn{2}{l}{$\begin{array}{l}\text { Buses where PMU is needed for observability of the radial buses } \\
\text { Ignoring ZIBs }\end{array}$} \\
\hline 14-bus & $B_{7}$ & Considering ZIBs \\
30-bus & $B_{9}, B_{12}, B_{25}$ & $B_{12}$ \\
39-bus & $B_{2}, B_{6}, B_{10}, B_{19}, B_{20}, B_{22}, B_{23}, B_{25}, B_{29}$ & $B_{10}, B_{20}, B_{23}, B_{25}, B_{29}$ \\
118-bus & $B_{9}, B_{12}, B_{68}, B_{71}, B_{86}, B_{110}$ & $B_{12}, B_{86}, B_{110}$ \\
$246-b u s$ & $B_{6}, B_{34}, B_{40}, B_{48}, B_{54}, B_{61}, B_{62}, B_{63}, B_{74}, B_{75}, B_{80}$, & $B_{6}, B_{34}, B_{40}, B_{48}, B_{106}, B_{125}, B_{132}, B_{158}, B_{168}$, \\
& $B_{106}, B_{122}, B_{125}, B_{126}, B_{132}, B_{147}, B_{158}, B_{168}, B_{181}$, & $B_{181}, B_{185}, B_{187}, B_{191}, B_{199}, B_{203}, B_{219}, B_{235}$ \\
& $B_{185}, B_{187}, B_{191}, B_{199}, B_{203}, B_{219}, B_{235}$ & \\
\hline
\end{tabular}

Note: '--' entry means none

Table 3. Optimal locations of PMUs for standard test system with computational time

\begin{tabular}{|c|c|c|c|c|c|c|}
\hline \multirow{2}{*}{$\begin{array}{l}\text { Test } \\
\text { system }\end{array}$} & \multicolumn{2}{|c|}{ Ignoring ZIBs } & \multicolumn{2}{|c|}{ Considering ZIBs } & \multirow[t]{2}{*}{ CPU (s) } & \multirow{2}{*}{$\begin{array}{l}\text { Elapsed } \\
\text { time (s) }\end{array}$} \\
\hline & $\begin{array}{l}\text { \# of } \\
\text { PMUs }\end{array}$ & Loc. of PMUs & $\begin{array}{l}\text { \# of } \\
\text { PMUs }\end{array}$ & Loc. of PMUs & & \\
\hline 14-bus & 04 & $B_{2}, B_{6}, B_{7}, B_{9}$ & 03 & $B_{2}, B_{6}, B_{9}$ & 0.171 & 0.133 \\
\hline 39-bus & 13 & $\begin{array}{l}B_{2}, B_{6}, B_{9}, B_{10}, B_{11}, B_{14}, B_{17}, B_{19}, B_{20} \\
B_{22}, B_{23}, B_{25}, B_{29}\end{array}$ & 08 & $B_{3}, B_{8}, B_{10}, B_{16}, B_{20}, B_{23}, B_{25}, B_{29}$ & 0.202 & 0.193 \\
\hline 118-bus & 32 & $\begin{array}{l}B_{2}, B_{5}, B_{9}, B_{12}, B_{15}, B_{17}, B_{21}, B_{23}, B_{28} \\
B_{34}, B_{37}, B_{40}, B_{45}, B_{49}, B_{52}, B_{56}, B_{62}, B_{63} \\
B_{68}, B_{71}, B_{75}, B_{77}, B_{80}, B_{85}, B_{90}, B_{94} \\
B_{101}, B_{105}, B_{110}, B_{114}\end{array}$ & 28 & $\begin{array}{l}B_{2}, B_{12}, B_{15}, B_{17}, B_{21}, B_{23}, B_{28}, B_{34}, \\
B_{37}, B_{40}, B_{45}, B_{49}, B_{52}, B_{62}, B_{63}, B_{68} \\
B_{71}, B_{75}, B_{77}, B_{80}, B_{85}, B_{90}, B_{94} \\
B_{101}, B_{105}, B_{110}, B_{114}\end{array}$ & 0.218 & 0.238 \\
\hline 246-bus & 70 & $\begin{array}{l}B_{3}, B_{6}, B_{7}, B_{10}, B_{11}, B_{18}, B_{21}, \\
B_{22}, B_{24}, B_{29}, B_{34}, B_{40}, B_{44}, B_{48}, B_{54}, B_{56}, \\
B_{57}, B_{61}, B_{62}, B_{65}, B_{70}, B_{74}, B_{75}, B_{78}, B_{80}, \\
B_{83}, B_{85}, B_{91}, B_{92}, B_{93}, B_{96}, B_{98}, B_{101}, \\
B_{106}, B_{109}, B_{113}, B_{117}, B_{121}, B_{125}, B_{128}, \\
B_{129}, B_{132}, B_{134}, B_{140}, B_{141}, B_{142}, B_{147}, \\
B_{157}, B_{158}, B_{160}, B_{163}, B_{168}, B_{173}, B_{181}, \\
B_{185}, B_{187}, B_{190}, B_{191}, B_{194}, B_{199}, B_{201}, \\
B_{202}, B_{203}, B_{215}, B_{216}, B_{219}, B_{229}, B_{234}, \\
B_{235}, B_{245}\end{array}$ & 62 & $\begin{array}{l}B_{3}, B_{6}, B_{7}, B_{10}, B_{11}, B_{18}, B_{21}, \\
B_{22}, B_{24}, B_{29}, B_{34}, B_{40}, B_{44}, B_{48}, B_{54}, \\
B_{56}, B_{57}, B_{65}, B_{78}, B_{80}, B_{83}, B_{85}, B_{91}, \\
B_{92}, B_{93}, B_{96}, B_{98}, B_{101}, B_{106}, B_{109}, \\
B_{113}, B_{117}, B_{121}, B_{125}, B_{128}, \\
B_{132}, B_{134}, B_{140}, B_{141}, B_{142}, \\
B_{157}, B_{158}, B_{160}, B_{163}, B_{168}, B_{173}, \\
B_{181}, B_{185}, B_{187}, B_{190}, B_{191}, B_{194}, \\
B_{199}, B_{201}, B_{202}, B_{203}, B_{219}, B_{229}, \\
B_{234}, B_{235}, B_{245}\end{array}$ & 0.374 & 0.468 \\
\hline
\end{tabular}

Table 4. Redundancy measurement for standard test system with ignoring ZIBs

\begin{tabular}{|c|c|c|c|}
\hline $\begin{array}{l}\text { Test } \\
\text { system }\end{array}$ & Bus observability index & $\begin{array}{l}\text { Max. } \\
\text { redundancy }\end{array}$ & TSORI \\
\hline 14-bus & $1,1,1,3,2,1,2,1,2,1,1,1,1,1$ & 3 & 19 \\
\hline 30-bus & $1,3,1,4,1,5,1,1,3,3,1,2,1,2,2,1,1,2,1,2,1,1,1,1,2,1,2,1,1,1$ & 5 & 50 \\
\hline 39-bus & $1,2,1,1,1,2,1,1,1,2,3,1,2,1,1,2,1,1,1,2,1,2,2,1,2,2,1,1,1,1,1,1,1,1,1,1,1$ & 3 & 49 \\
\hline 118-bus & $\begin{array}{l}1,2,2,1,1,1,1,2,1,1,2,2,1,2,2,2,2,1,2,1,1,2,1,1,1,1,1,1,1,1,1,1,2,1,1,1,3,1,2, \\
2,1,2,1,1,2,1,1,1,1,1,2,1,1,2,1,1,1,1,2,1,1,1,1,1,1,2,1,1,2,2,1,1,1,1,2,1,3,1, \\
1,2,2,1,1,1,1,1,1,1,2,1,1,1,1,1,1,2,1,1,1,1,1,1,2,1,1,1,1,1,1,1,1,1,1,1,1,1,1,1\end{array}$ & 3 & 150 \\
\hline 246-bus & $\begin{array}{l}2,1,1,1,1,2,2,2,1,1,1,1,1,1,1,3,3,2,1,2,2,2,1,2,2,1,2,1,1,1,1,1,1,2,2,1,2,1,1, \\
1,1,1,1,4,2,3,1,1,1,1,1,1,1,2,2,3,2,1,2,1,1,2,1,1,3,1,3,1,2,1,3,1,2,1,2,1,1,2, \\
1,3,1,3,1,1,1,3,1,1,1,2,3,1,1,1,2,2,2,1,1,1,2,1,2,2,2,1,1,1,3,2,2,1,2,2,1,2,1 \\
1,2,1,3,1,1,1,1,2,2,1,2,2,2,3,1,3,1,3,1,1,1,2,1,1,1,1,1,1,2,1,1,2,1,1,1,3,1,1 \\
2,4,1,3,1,3,1,2,1,2,1,1,1,1,1,1,1,1,1,1,1,1,1,1,2,1,2,1,2,1,2,1,1,1,3,1,1,1,1 \\
1,1,2,1,2,1,2,2,2,1,1,1,1,1,1,1,1,3,1,1,1,1,1,2,1,1,1,1,1,1,1,1,1,2,2,1,2,2,1, \\
1,1,1,2,3,1,1,1,1,1,1,1\end{array}$ & 4 & 360 \\
\hline
\end{tabular}

Table 5. Redundancy measurement for standard test system with considering ZIBs

\begin{tabular}{|c|c|c|c|}
\hline $\begin{array}{l}\text { Test } \\
\text { system }\end{array}$ & Bus observability index & $\begin{array}{l}\text { Max. } \\
\text { redundancy }\end{array}$ & TSORI \\
\hline 14-bus & $1,1,1,2,2,1,1,1,1,1,1,1,1,1$ & 2 & 16 \\
\hline 30-bus & $1,1,1,2,1,1,1,1,1,1,1,1,1,1,1,1,1,1,1,1,1,1,1,1,1,1,1,1,1,1$ & 2 & 31 \\
\hline 39-bus & $1,1,1,1,1,1,1,1,1,1,1,1,1,1,1,1,1,1,1,1,1,1,1,2,1,2,1,1,1,1,1,1,1,1,1,1,1,1,1$ & 2 & 41 \\
\hline 118-bus & $\begin{array}{l}1,2,2,1,1,1,1,1,1,1,1,2,1,2,2,2,2,1,2,1,1,2,1,1,1,1,1,1,1,1,1,1,2,1,1,1,3,1,2,2, \\
1,2,1,1,2,1,1,1,1,1,2,1,1,1,1,1,1,1,1,1,1,1,1,1,1,2,1,1,2,2,1,1,1,1,2,1,3,1,1,2, \\
2,1,1,1,1,1,1,1,2,1,1,1,1,1,1,2,1,1,1,1,1,1,2,1,1,1,1,1,1,1,1,1,1,1,1,1,1,1\end{array}$ & 3 & 146 \\
\hline 246-bus & $\begin{array}{l}2,1,1,1,1,2,2,2,1,1,1,1,1,1,1,3,3,2,1,2,2,2,1,2,2,1,1,1,1,1,1,1,1,2,2,1,2,1,1,1, \\
1,1,1,4,2,3,1,1,1,1,1,1,1,1,2,3,2,1,1,1,1,1,1,1,3,1,3,1,1,1,2,1,1,1,1,1,1,2,1,3, \\
1,3,1,1,1,2,1,1,1,2,2,1,1,1,2,2,2,1,1,1,2,1,2,1,2,1,1,1,3,2,2,1,1,2,1,2,1,1,2,1, \\
3,1,1,1,1,1,1,1,1,2,2,3,1,2,1,3,1,1,1,2,1,1,1,1,1,1,1,1,1,1,1,1,1,2,1,1,2,4,1,3, \\
1,3,1,2,1,2,1,1,1,1,1,1,1,1,1,1,1,1,1,1,2,1,2,1,2,1,2,1,1,1,3,1,1,1,1,1,1,2,1,2, \\
1,2,2,1,1,1,1,1,1,1,1,1,2,1,1,1,1,1,2,1,1,1,1,1,1,1,1,1,2,2,1,1,2,1,1,1,1,1,2,1,\end{array}$ & 4 & 336 \\
\hline
\end{tabular}


In the present work, redundancy measurement is also more systematically assigned. For example, in the case of IEEE 14-bus the maximum redundancy for a bus is 3 (in case of ignoring ZIBs) and 2 (in case of considering ZIBs). The optimal number of PMU obtained using the proposed approach has been compared with the existing standard results found in the literature are shown in Table 6. It can be observed that the optimal number of PMUs necessary to make the interconnected power network (IEEE 14.30,118, New England 39, and NRPG 246-bus system) fully observable are somewhat the same as the results published in the open literature.

Table 6. Comparison of results with the existing standard approach under usual operating states

\begin{tabular}{llllll}
\hline Test system & 14-bus & 30 -bus & 39 -bus & 118 -bus & 246-bus \\
\hline S. Chakrabarti and E. Kyriakides [10] & 4 & 10 & 13 & -- & -- \\
T. Venkatesh and T. Jain [12] & 4 & 10 & - & -- & 83 \\
Roy et al. [15] & 4 & 10 & 13 & 32 & -- \\
S. Mousavian and M. J. Feizollahi [16] & 4 & 10 & 13 & 32 & - \\
Sodhi et al. [17] & 7 & -- & 26 & -- & 135 \\
B. Xu and A. Abur [21] & 4 & 10 & -- & 32 & -- \\
B. Gou [24] & 4 & -- & -- & -- & - \\
Proposed approach & $\mathbf{4}$ & $\mathbf{1 0}$ & $\mathbf{1 3}$ & $\mathbf{3 2}$ & $\mathbf{7 0}$ \\
\hline Note. '-' entry mens not reported & & & & &
\end{tabular}

\section{CONCLUSION}

This paper consummated two goals. Firstly, an ILP based approach for finding optimal locations of PMU is explored and secondly, the explored approach is executed and tested on several standard test systems. The first step in placing the PMUs is the identification of a candidate location. In a power system, there may be certain buses that are strategically important, so that a PMU must be located at each of those buses. The remaining buses are made observable by installing a minimum number of additional PMUs. The radial buses are excluded from the list of potential locations for placing a PMU. As PMU placed at a radial bus can measure the voltage phasors at that bus and only one additional bus that is associated with it. Further, PMU placed at the bus connected to the radial bus can measure the voltage phasor of the radial bus by using the measurement of the current phasor through the radial line. Therefore, a PMU is pre-assigned to each bus connected to a radial bus. Pre-assigning PMUs to certain buses in this manner, the system observability is satisfied since no violation constraint exists. Thus, the number of PMUs are the same for each power system since the system observability is satisfied with the improvement that no radial buses are included in the optimal solutions. The offering of the paper remains in benchmarking the universal OPP solutions for a number of extensively used systems and determine the optimal locations of PMUs for the system. The obtained results indicate the efficacy of the proposed approach of OPP for interconnected power network observability. After comparing with the standard approaches in the earlier published literature, the simulation results show that the proposed approach determines the minimum number of PMUs, unlike earlier approaches which may find either the same or even higher number of PMUs.

\section{REFERENCES}

[1] T. L. Baldwin, L. Mili, M. B. Boisen, and R. Adapa, "Power system observability with minimal phasor measurement placement," IEEE Transactions on Power Systems, vol. 8, no. 2, pp. 707-715, May 1993.

[2] A. G. Phadke, "Synchronized phasor measurements in power systems," IEEE Computer Applications in Power, vol. 6, no. 2, pp. 10-15, April 1993.

[3] A. G. Phadke, "Synchronized sampling and phasor measurements for relaying and control," IEEE Transactions on Power Delivery, vol. 9, no. 1, pp. 442-452, Jan. 1994.

[4] A. G. Phadke and J. S. Thorp, "Synchronized phasor measurements and their applications," Springer US, 2008.

[5] A. Monticelli and F. F. Wu, "Network observability: Identification of observable islands and measurement placement," IEEE Power Engineering Review, vol. PER-5, no. 5, pp. 32-32, May 1985.

[6] A. Monticelli, "State estimation in electric power systems: A generalized approach," Springer US, 1999.

[7] A. Abur and A. G. Expósito, "Power system state estimation: Theory and implementation," CRC Press, 2004.

[8] A. G. Phadke, J. S. Thorp and K. J. Karimi, "State estimation with phasor measurements," IEEE Transactions on Power Systems, vol. 1, no. 1, pp. 233-238, Feb. 1986.

[9] S. Chakrabarti and E. Kyriakides, "Optimal placement of phasor measurement units for state estimation," IASTED International Conference on Power Energy Systems, EuroPES, Spain, January 2007.

[10] S. Chakrabarti and E. Kyriakides, "Optimal Placement of Phasor Measurement Units for Power System Observability in IEEE Transactions on Power Systems, vol. 23, no. 3, pp. 1433-1440, Aug. 2008.

[11] R. Babu and B. Bhattacharyya, "Optimal allocation of phasor measurement unit for full observability of the connected power network," International Journal of Electrical Power \& Energy Systems, vol. 79, pp. 89-97, July 2016. 
[12] V. Thirugnanasambandam and T. Jain, "Intelligent-search technique based strategic placement of synchronized measurements for power system observability," Expert Systems with Applications, vol. 42, no. 10, pp. 4768-4777, 2015.

[13] F. J. Marin, F. Garcia-Lagos, G. Joya, and F. Sandoval, "Genetic algorithms for optimal placement of phasor measurement units in electrical networks," Electronics Letters, vol. 39, no. 19, pp. 1403-1405, Sept. 2003.

[14] B. K. S. Roy, A. K. Sinha, and A. K. Pradhan, "Optimal phasor measurement unit placement for power system observability - A heuristic approach," 2011 IEEE Symposium on Computational Intelligence Applications In Smart Grid (CIASG), Paris, pp. 1-6, 2011.

[15] B. K. S. Roy, A. K. Sinha, and A. K. Pradhan, "An optimal PMU placement technique for power system observability," International Journal of Electrical Power \& Energy Systems, vol. 42, no. 1, pp. 71-77, Nov. 2012.

[16] S. Mousavian and M. J. Feizollah, "An investment decision model for the optimal placement of phasor measurement units," Expert Systems with Applications, vol. 42, no. 21, pp. 7276-7284, Nov. 2015.

[17] R. Sodhi, S. C. Srivastava, and S. N. Singh, "Multi-criteria decision-making approach for multistage optimal placement of phasor measurement units," IET Generation, Transmission \& Distribution, vol. 5, no. 2, pp. 181-190, 2011.

[18] K. Jamuna and K. S. Swarup, "Optimal placement of PMU and SCADA measurements for security constrained state estimation," International Journal of Electrical Power \& Energy Systems, vol. 33, no. 10, pp. 1658-1665, 2011.

[19] K. Jamuna and K. S. Swarup, "Multi-objective biogeography based optimization for optimal PMU placement," Applied Soft Computing, vol. 12, no. 5, pp. 1503-1510, May 2012.

[20] B. Xu and A. Abur, "Observability analysis and measurement placement for system with PMUs," IEEE PES Power Systems Conference and Exposition, 2004, New York, NY, vol. 2, pp. 943-946, 2004.

[21] B. Xu and A. Abur, "Optimal placement of phasor measurement units for state estimation," PSERC Publication, Texas A\&M University, Oct. 2005.

[22] R. Kavasseri and S. K. Srinivasan, "Joint placement of phasor and conventional power flow measurements for fault observability of power systems," IET Generation, Transmission \& Distribution, vol. 5, no. 10, pp. 1019-1024, 2011.

[23] R. Kavasseri and S. K. Srinivasan, "Joint placement of phasor and power flow measurements for observability of power systems," IET Generation, Transmission \& Distribution, vol. 5, no. 10, pp. 1019-1024, Oct. 2011.

[24] B. Gou, "Optimal Placement of PMUs by Integer Linear Programming," IEEE Transactions on Power Systems, vol. 26, no. 4, pp. 1929-1936, Nov. 2011.

[25] B. Gou, "Generalized integer linear programming formulation for optimal PMU placement," IEEE Transactions on Power Systems, vol. 23, no. 3, pp. 1525-1526, Aug. 2008.

[26] F. Rashidia, E. Abiri, T. Niknamb, and M. R. Salehia, "Optimal placement of PMUs with limited number of channels for complete topological observability of power systems under various contingencies," International Journal of Electrical Power \& Energy Systems, vol. 67, pp. 125-137, May 2015.

[27] X. Tai, D. Marelli, E. Rohr, and M. Fu, "Optimal PMU placement for power system state estimation with random component outages," International Journal of Electrical Power \& Energy Systems, vol. 51, pp. 35-42, Oct. 2013.

[28] L. Sun, T. Chen, X. Chen, W. K. Ho, K. Ling, K. Tseng, and G. A. J. Amaratunga, "Optimum placement of phasor measurement units in power systems," IEEE Trans. on Inst. and Measurement, vol. 68, no. 2, pp. 421-429, 2019.

[29] N. M. Manousakis and G. N. Korres, "Optimal Allocation of phasor measurement units considering various contingencies and measurement redundancy," IEEE Transactions on Instrumentation and Measurement, pp. 1-1, 2019.

[30] A. Almunif and L. Fan, "Optimal PMU placement for modeling power grid observability with mathematical programming methods," International Transactions on Electrical Energy Systems, vol. 30, no. 2, Aug. 2019.

[31] S. Chatterjee, P. K. Ghosh, and B. K. S. Roy, "PMU-based power system component monitoring scheme satisfying complete observability with multicriteria decision support," International Transactions on Electrical Energy Systems, vol. 30, no. 2, Oct. 2019.

[32] Y. Matsukawa, M. Watanabe, Y. Mitani, and M. L. Othman, "Multi-objective PMU placement optimization considering the placement cost including the current channel allocation and state estimation accuracy," IEEJ Transactions on Power and Energy, vol. 139, no. 2, pp. 84-90, Feb. 2019.

[33] N. P. Theodorakatos, "Optimal phasor measurement unit placement for numerical observability using a two-phase branch-and-bound algorithm," International Journal of Emerging Electric Power Systems, vol. 19, no. 3, 2018.

[34] N. P. Theodorakatos, "Optimal phasor measurement unit placement for numerical observability using branch-and-bound and a binary-coded genetic algorithm," Electric Power Components and Systems, vol. 47, no. 4-5, pp. 357-371, 2019.

[35] "Power systems test case archive," Department of Electrical Engineering, University of Washington, 1979. [Online]. Available at: https://www2.ee.washington.edu/research/pstca/.

[36] P. Ledesma, "New England test system, IEEE 39 Bus System, 10 generators, in PSS/E format (version 29)," [Online]. Available at: http://electrica.uc3m.es/pablole/new_england.html

[37] "Northern regional power grid (NRPG) data - IIT Kanpur," Department of Electrical Engineering, IIT Kanpur, [Online]. Available at: https://www.iitk.ac.in/eeold/facilities/Research_labs/Power.../NRPG-DATA.pdf.

[38] F. Aminifar, C. Lucas, A. Khodaei, and M. Fotuhi-Firuzabad, "Optimal placement of phasor measurement units using immunity genetic algorithm," IEEE Transactions on Power Delivery, vol. 24, no. 3, pp. 1014-1020, 2009.

[39] R. Babu and B. Bhattacharyya, "Strategic placements of PMUs for power network observability considering redundancy measurement," Measurement, vol. 134, pp. 606-623, Feb. 2019.

[40] M. T. Mouwafi, R. A. El-Sehiemy, A. A. El-Ela, and A. M. Kinawy, "Optimal placement of phasor measurement units with minimum availability of measuring channels in smart power systems," Electric Power Systems Research, vol. 141, pp. 421-431, Dec. 2016.

[41] TOMLAB, "The TOMLAB optimization environment," TOMLAB®, [Online]. Available at: https://tomopt.com/tomlab/.

[42] TOMLAB/MINLP manual, "TOMLAB optimization," [Online]. Available at: https://tomopt.com/docs/TOMLAB_QUICKGUIDE.pdf.

\footnotetext{
Optimal placement of PMU for complete observability of the interconnected power network... (Rohit Babu)
} 


\section{BIOGRAPHIES OF AUTHORS}
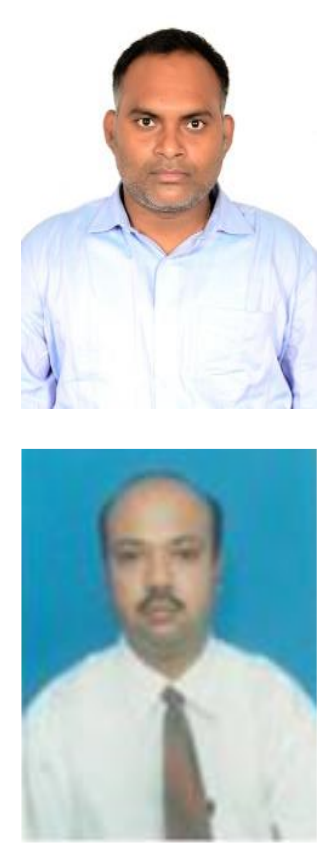

Rohit Babu was born in Mirzapur, India in 1986. He is currently working as an Assistant Professor of Electrical and Electronics Engineering Department, Bharat Institute of Engineering and Technology, Hyderabad (India). He is also currently pursuing his Ph.D. degree at the Indian Institute of Technology (Indian School of Mines), Dhanbad (India). He had served the Department of Electrical and Electronics Engineering of Raffles University, Rajasthan (India) for one year as an Assistant Professor. He received B.Tech. degree from United College of Engineering \& Research, Greater Noida (India) in 2011 and M.Tech. Degree from Indian Institute of Technology (Banaras Hindu University), Varanasi (India) in 2013. His research interests include power system observability, optimal PMU placement, power system optimization and state estimation.

Biplab Bhattacharyya is born on 9th January 1970. He is currently working as an Associate Professor of Electrical Engineering at the Indian Institute of Technology (Indian School of Mines), Dhanbad, India. He has joined the electrical engineering department as assistant professor in the year 2007. Then he promoted to the post of associate professor in 2010. He had served the Department of Electrical Engineering of National Institute of Technology, Durgapur, India for six years as senior lecturer. He was in the position of lecturer in the Electrical Engineering Department of BITS, Pilani, Rajasthan, India for nearly one year. He worked as assistant engineer (electrical test), in the reputed cable industry for nearly three years. He obtained B.Sc (Hons) in physics from Calcutta University, India in 1990. He obtained his B-Tech and M-Tech degree in the field of electrical machines and power systems from Calcutta University in 1993 and 1995, respectively. He obtained his Ph.D. degree in engineering from the Department of Electrical Engineering, Jadavpur University, India in 2006. He has published several technical papers in international/national journals and conference proceedings. His research area mainly includes evolutionary approaches, power system optimization, planning, dispatch, FACTS devices. 